\title{
ReaR
}

ISNN 19894090

Revista electrónica de AnestesiaR

Febrero 2021

FORMACIÓN MÉDICA

\section{Por tus acciones te juzgarán. Cálculo del tamaño de la muestra en estudios de supervivencia.}

Molina Arias M.

\section{Hospital Infantil Universitario La Paz, Madrid}

\section{Resumen}

El cálculo del tamaño muestral estima el número de participantes que serán necesarios para demostrar el efecto considerado como clínicamente importante, si este existe. En el caso de los estudios de supervivencia cobra más importancia el cálculo del número de sucesos que es preciso observar, a partir del cual se calculará el número de participantes necesarios.

Introducción

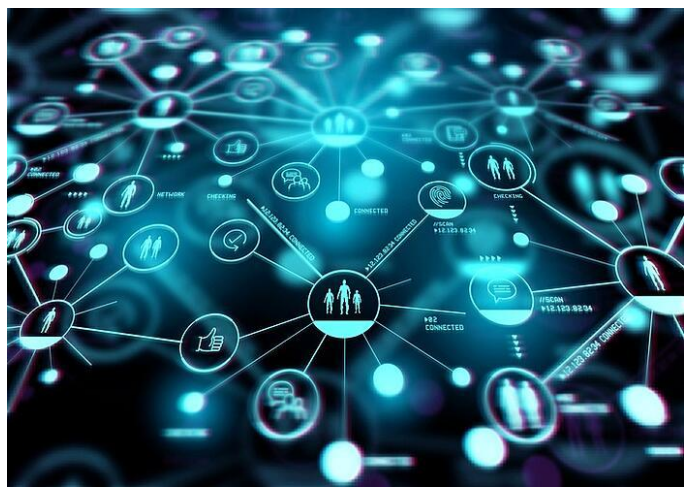

El cálculo del tamaño muestral estima el número de participantes que serán necesarios para demostrar el efecto considerado como clínicamente importante, si este existe. En el caso de los estudios de supervivencia cobra más importancia el cálculo del número de sucesos que es preciso observar, a partir del cual se calculará el número de participantes necesarios.

Hoy me vais a perdonar, pero vengo un poco bíblico. $\mathrm{Y}$ es que estaba pensando en el cálculo de la muestra para los estudios de supervivencia $y$ me ha recordado el mensaje que nos transmite
Ezequiel: según tus caminos y tus obras te juzgarán.

Una vez más, pensaréis que de tanto darle vueltas a la medicina basada en la evidencia se me ha ido un poco la cabeza, pero si aguantáis un poco y seguís leyendo, veréis que la analogía tiene su punto.

Uno de los indicadores de calidad metodológica más valorados de un estudio es el cálculo previo del tamaño muestral necesario para demostrar (o rechazar) la hipótesis de trabajo. Cuando queremos estudiar el efecto de una intervención debemos, a priori, definir qué tamaño de efecto queremos detectar y calcular el tamaño muestral necesario para poder hacerlo, siempre que el efecto exista (algo que deseamos cuando planteamos el experimento, pero que desconocemos a priori), teniendo en cuenta para ello el nivel de significación $\mathrm{y}$ la potencia que queramos que tenga el estudio.

En resumen, si detectamos el tamaño de efecto que previamente establecimos, la diferencia entre los dos grupos será estadísticamente significativa (nuestra 
ansiada $\mathrm{p}<0,05)$. Por el contrario, si no hay diferencia significativa, probablemente no exista diferencia real, aunque siempre con el riesgo de cometer un error de tipo 2 que es igual a 1 menos la potencia del estudio.

Hasta aquí parece que está claro, tenemos que calcular el número de participantes que necesitamos. Pero esto no es tan sencillo para los estudios de supervivencia.

Los estudios de supervivencia agrupan una serie de técnicas estadísticas para aquellas situaciones en las que no basta observar un suceso, sino que es fundamental el tiempo que transcurre hasta que el suceso se produce. En estos casos, la variable de resultado no será ni cuantitativa ni cualitativa, sino de tiempo a suceso. Es una especia de variable mixta que tendría una parte dicotómica (el suceso se produce o no) y una cuantitativa (cuánto tarda en producirse).

El nombre de estudios de supervivencia es un poco engañoso y uno puede pensar que el suceso en estudio será la muerte de los participantes, pero nada más lejos de la realidad. El suceso puede ser cualquier tipo de evento, bueno o malo para el participante. Lo que ocurre es que los primeros estudios se aplicaron a situaciones en los que el suceso de interés era la muerte y el nombre ha prevalecido.

En estos estudios, el periodo de seguimiento de los participantes suele ser desigual e, incluso, algunos pueden terminar el estudio sin presentar el suceso de interés o perderse del estudio antes de que finalice.

Por estos motivos, si queremos saber si hay diferencias entre la presentación del suceso de interés en las dos ramas del estudio, para calcular la muestra no será tan importante el número de sujetos de participen, sino el número de sucesos que necesitamos para que la diferencia sea significativa si se alcanza la diferencia clínicamente importante, que deberemos establecer a priori.

Vamos a ver cómo se hace, dependiendo del tipo de contraste que tengamos pensado utilizar.

Si solo queremos determinar el número de sucesos necesarios que tenemos que observar para detectar una diferencia entre un grupo determinado y la población de la que procede, la fórmula para hacerlo es la siguiente:

$$
E=\frac{2 K}{(\ln T R)^{2}}
$$

Donde $\mathrm{E}$ es el número de sucesos que necesitamos observar, $\mathrm{K}$ es el valor determinado por el nivel de confianza y la potencia del estudio y $\operatorname{lnTR}$ es el logaritmo natural de la tasa de riesgo.

El valor de $K$ se calcula como $\left(Z_{\alpha}+\right.$ $\left.Z_{\beta}\right)^{2}$, siendo $\mathrm{z}$ el valor estandarizado para el nivel de confianza y de potencia elegido. Lo más habitual es realizar un contraste bilateral (con dos colas) con un nivel de confianza de 0,05 y una potencia de $80 \%$. En este caso, los valores son $Z_{\alpha}=1,96, Z_{\beta}=0,84$ y $K=$ 7,9. En la tabla adjunta os dejo los valores más frecuentes de $\mathrm{K}$, para que no tengáis que calcularlos.

\begin{tabular}{|c|c|c|c|c|c|}
\hline \multirow{2}{*}{$\begin{array}{c}\text { Contraste } \\
\text { bilateral }\end{array}$} & \multicolumn{4}{|c|}{ Potencia del estudio } & $\begin{array}{c}\text { Contraste } \\
\text { unilateral }\end{array}$ \\
\hline & $50 \%$ & $80 \%$ & $90 \%$ & $95 \%$ & 0,05 \\
\hline 0,1 & 2,7 & 6,2 & 8,6 & 10,8 & 0,25 \\
\hline 0,05 & 3,8 & 7,9 & 10,5 & 13,0 & 0,01 \\
\hline 0,025 & 5,4 & 10,0 & 13,0 & 15,8 & 0,017 \\
\hline 0,01 & 6,6 & 11,7 & 14,9 & 17,8 & 0,005 \\
\hline
\end{tabular}

Cálculo del valor de $\mathrm{K}$ según el nivel de confianza, el tipo de contraste y la potencia del estudio.

$\mathrm{K}=(\mathrm{Z} \alpha+\mathrm{Z} \beta) 2$ 
La tasa de riesgo es el cociente entre el riesgo del grupo en estudio y el riesgo en la población, que se supone que conocemos. Se define como $\mathrm{Sm}_{1} / \mathrm{Sm}_{2}$, donde $\mathrm{Sm}_{1}$ es el tiempo medio de aparición del evento en la población y $\mathrm{Sm}_{2}$ el que esperamos en el grupo de estudio.

Vamos a poner un ejemplo para entender mejor lo dicho hasta ahora.

Supongamos que los pacientes que toman un determinado fármaco (que llamaremos A para no esforzarnos mucho) tienen riesgo de desarrollar úlcera de estómago durante el primer año de tratamiento. Ahora seleccionamos un grupo y les damos un tratamiento (B, esta vez) que actúa como profilaxis, de tal forma que esperamos que el evento tarde un año más en producirse. ¿Cuántas úlceras tenemos que observar para un estudio con un nivel de confianza de 0,05 y una potencia de $0,8(80 \%)$ ?

$\mathrm{K}$ sabemos que vale 7,9. $\mathrm{Sm}_{1}=1 \mathrm{y}$ $\mathrm{Sm}_{2}=2$. Sustituimos sus valores en la fórmula que ya conocemos:

$$
E=\frac{2 K}{(\ln T R)^{2}}=\frac{2 \times 7,9}{(\ln 1 / 2)^{2}}=\frac{15,8}{0,48}=32,9
$$

Necesitaremos observar 33 úlceras durante el seguimiento. Ahora ya podemos calcular cuántos pacientes tenemos que incluir en el estudio (veo complicado reclutar úlceras).

Vamos a suponer que podemos reclutar 12 pacientes anuales. Si tenemos que observar 33 úlceras, el seguimiento se deberá prolongar durante $33 / 12=2,75$, o sea, 3 años. Para más seguridad, planificaríamos un seguimiento un poco superior.

Este es el caso más sencillo. Cuando lo que queremos es comparar las dos curvas de supervivencia (pensamos hacer una prueba de log-rank), el cálculo del tamaño muestral es un pelín más complejo, pero no mucho. Al fin y al cabo, ya estaremos comparando las curvas de probabilidad de supervivencia de los dos grupos.

En estos casos, la fórmula para el cálculo del número de sucesos necesarios es la siguiente:

$$
E=\frac{(C T R+1)^{2}(K)}{C(T R-1)^{2}}
$$

Nos encontramos un parámetro nuevo, $\mathrm{C}$, que es la relación de participantes entre un grupo y el otro (1:1, 1:2, etc.).

Pero hay otra diferencia con el supuesto anterior. En estos casos la TR se calcula como el cociente de los logaritmos naturales de $\pi_{1} \mathrm{y} \pi_{2}$, que son las proporciones de participantes de cada grupo que presentan el evento en un periodo de tiempo determinado.

Siguiendo el ejemplo anterior, supongamos que conocemos que el riesgo de úlcera en los que toman $\mathrm{A}$ es del $50 \%$ en los 6 primeros meses y el de los que toman $\mathrm{B}$ del 20\%. ¿Cuántas úlceras necesitamos observar con el mismo nivel de confianza y la misma potencia del estudio?

Sustituyamos los valores en la fórmula anterior:

$$
E=\frac{(C T R+1)^{2}(K)}{C(T R-1)^{2}}=\frac{(1 \times(\ln 0,2 / \ln 0,5)+1)^{2} \times 7,9}{1 \times((\ln 0,2 / \ln 0,5)-1)^{2}}=\frac{87,18}{1,747}=49,9
$$

Necesitaremos observar 50 úlceras durante el estudio. Ahora necesitamos saber cuántos participantes (no sucesos) necesitamos en cada rama del estudio. 
Lo podemos obtener con la siguiente fórmula:

$$
n=\frac{E}{2-\left(\pi_{1}-\pi_{2}\right)}
$$

Si sustituimos nuestros valores en la ecuación, obtenemos un valor de 29,4, así que necesitaremos 30 pacientes en cada rama del estudio, 60 en total.

Para ir terminando ya, vamos a ver qué pasaría si queremos una proporción de participantes diferente de la más fácil, 1:1. En ese caso, el cálculo de $n$ con la última fórmula debe ajustarse teniendo en cuenta esta proporción, que es nuestra conocida $\mathrm{C}$ :

$$
n=\frac{C E}{\left\{\left(1-\pi_{1}\right)+C\left(1-\pi_{2}\right)\right\}^{2}}
$$

Supongamos que queremos una relación $2: 1$. Sustituimos los valores en la ecuación:

$$
n=\frac{C E}{\left\{\left(1-\pi_{1}\right)+C\left(1-\pi_{2}\right)\right\}^{2}}=\frac{2 \times 50}{\{(1-0,5)+2(1-0,2)\}^{2}}=22,6
$$

Necesitaríamos 23 participantes en una rama y 46, el doble, en la otra, 69 en total.

Y aquí lo dejamos por hoy. Como siempre, todo lo que hemos dicho en esta entrada es para que podamos comprender los fundamentos del cálculo de la muestra necesaria en este tipo de estudios, pero os aconsejo que, si tenéis que hacerlo alguna vez, utilicéis un programa estadístico o una calculadora de tamaño muestral. Hay muchas disponibles y algunas hasta son totalmente gratis.

Espero que ahora comprendáis lo de Ezequiel y que, en estos estudios, son más importantes las cosas que hacemos (o padecemos) que cuántos las hacemos (o padecemos). Hemos vista la forma más sencilla para calcular el tamaño de la muestra de un estudio de supervivencia, aunque podríamos complicarnos la vida y calcular el tamaño muestral basándonos en estimaciones de los riesgos relativos o de las tasas de riesgos instantáneos, nuestras queridas hazard ratios. Pero esa es otra historia...

\section{Bibliografía}

- Cálculo del tamaño de muestra. Estudios para el análisis de sobrevida en un grupo. En: Velasco Rodríguez VM, Martínez Ordaz VA, Roiz Hernández J, Huazano García F, Nieves Rentería A, eds. Muestreo y tamaño e muestra. Una guía práctica para personal de salud que realiza investigación. E-libro.net, Torreón, Coahuila, México, 2002: 79-81. ( $\underline{\text { PDF }}$ )

- Marrugat J, Vila J, Pavesi M, Sanz F. Estimación del tamaño de la muestra en la investigación clínica y epidemiológica. Med Clin (Barc).1998;111:267-76. (PDF)

Correspondencia al autor

Manuel Molina Arias mma1961@gmail.com

Servicio de Gastroenterología.

Hospital Infantil Universitario La Paz. Madrid. España.

Aceptado para el blog en julio de 2020 